University of Nebraska - Lincoln

DigitalCommons@University of Nebraska - Lincoln

9-1-1999

\title{
Quasicoherent nucleation mode in two-phase nanomagnets
}

\author{
Ralph Skomski \\ University of Nebraska-Lincoln, rskomski2@unl.edu \\ J. Ping Liu \\ University of Nebraska-Lincoln, pliu@uta.edu \\ David J. Sellmyer \\ University of Nebraska-Lincoln, dsellmyer@unl.edu
}

Follow this and additional works at: https://digitalcommons.unl.edu/physicssellmyer

Part of the Physics Commons

Skomski, Ralph; Liu, J. Ping; and Sellmyer, David J., "Quasicoherent nucleation mode in two-phase nanomagnets" (1999). David Sellmyer Publications. 77.

https://digitalcommons.unl.edu/physicssellmyer/77

This Article is brought to you for free and open access by the Research Papers in Physics and Astronomy at DigitalCommons@University of Nebraska - Lincoln. It has been accepted for inclusion in David Sellmyer Publications by an authorized administrator of DigitalCommons@University of Nebraska - Lincoln. 


\title{
Quasicoherent nucleation mode in two-phase nanomagnets
}

\author{
Ralph Skomski,* J. P. Liu, and D. J. Sellmyer \\ Behlen Laboratory of Physics and Center for Materials Research and Analysis, University of Nebraska, Lincoln, Nebraska 68588-0111
}

(Received 17 March 1999)

\begin{abstract}
Magnetization processes in advanced magnetic nanostructures are investigated. For the case of spherical soft or semihard grains surrounded by a very hard matrix a bulging nucleation mode is discovered. The bulging mode exhibits the radial angular symmetry of the coherent mode, but it is incoherent due to its radial variation. The radial dependence of the bulging mode is obtained by solving a spherical Bessel equation which is subject to appropriate boundary conditions. In contrast to the coherent mode, the bulging mode yields a nucleationfield coercivity which depends on the exchange stiffness and on the size of the grain. There is a critical grain radius $7.869 \sqrt{A / \mu_{0} M_{s}^{2}}$ above which the bulging mode is replaced by a modified curling mode. The nucleation modes realized in nanostructures affect the demagnetizing-field corrections necessary to account for the external shape of magnetic samples. Since strong but short-range exchange and weak but long-range magnetostatic interactions compete on nanostructural length scales, the sample-shape dependence of the hysteresis loops cannot be mapped onto a purely magnetostatic demagnetizing factor. [S0163-1829(99)01734-8]
\end{abstract}

\section{INTRODUCTION}

Nanostructured magnets are of great interest in theoretical physics, solid-state science, and advanced technological areas such as permanent magnetism and magnetic recording. ${ }^{1-6}$ From a practical point of view, the number of pure compounds meeting specific magnetic requirements is limited, but the magnetic performance of two-phase structures may be better than that of the single-phase magnets. This refers in particular to the energy product $(B H)_{\max }$, which describes the amount of magnetostatic energy stored by a permanent magnet. ${ }^{1}$ An enhancement of the maximum energy products beyond those of hard-magnetic phases such as $\mathrm{SmCo}_{5}$ and $\mathrm{PtFe}$ is possible by exchange coupling nanostructured soft regions having a high magnetization, such as $\mathrm{Fe}_{65} \mathrm{Co}_{35}$, to a highly anisotropic and coercive hard matrix. On this basis, room-temperature energy products as high as about $400 \mathrm{~kJ} / \mathrm{m}^{3}$ (50 MG Oe) have recently been obtained in iron-rich two-phase Pt-Fe thin films. ${ }^{5}$ This energy product is close to energy products of the present record-holder $\mathrm{Nd}_{2} \mathrm{Fe}_{14} \mathrm{~B}$ and clearly exceeds energy products achieved in single-phase PtFe films. Taking into account the comparatively poor performance of the starting material PtFe, this result is a clear confirmation of the theoretical prediction ${ }^{1}$ of enhanced energy products in suitable nanostructures.

A key theoretical problem in micromagnetism is to calculate the hysteresis loops of two-phase materials from the magnet's morphology, that is from its microstructure and nanostructure. Hard-magnetic hysteresis is associated with low-temperature anisotropy-energy minima, ${ }^{2,6}$ as opposed, e.g., to metastabilities in the vicinity of the critical point ${ }^{7}$ and processes involving variable electric fields in soft magnets. ${ }^{8}$ For ideally aligned two-phase magnets analytic expressions for extrinsic properties such as the energy product $(B H)_{\max }$ have been obtained as a function of the spatial distribution of the first anisotropy constant $K_{1}(\mathbf{r}) .{ }^{1}$ In particular, when the radius of the soft regions is smaller than the domain wallwidth of the hard phase, then the calculation reduces to the consideration of the volume-averaged anisotropy constant $\left\langle K_{1}\right\rangle$. In the case of $\mathrm{PtFe}$, this regime corresponds to soft inclusions smaller than about $8 \mathrm{~nm}$, and TEM micrographs show indeed that many soft grains are much smaller than 10 $\mathrm{nm} .{ }^{5}$ However, there are also soft grains (and clusters of soft grains) larger than $20 \mathrm{~nm}$. These extended soft regions have a disproportionally strong influence on the hysteresis loop but cannot be described in terms of $\left\langle K_{1}\right\rangle$ exclusively. ${ }^{9}$

Magnetization processes in inhomogeneous magnets are generally very complicated. ${ }^{8,10}$ Often it is possible to use approximations to investigate the physics of magnetization processes (see, e.g., Refs. 2,6,9,11-13), but quantitative results are usually obtained from numerical calculations. ${ }^{14-18}$ By comparison, analytic approaches have been limited to a few simple, mostly homogeneous geometries. $1,10,13,15-17,19-25$

The determination of the local magnetization configuration $\mathbf{M}(\mathbf{r})$ starts from the well-known micromagnetic energy functional

$$
E=\int\left[A \frac{(\nabla \mathbf{M})^{2}}{M_{s}^{2}}-K_{1}(\mathbf{r}) \frac{M_{z}^{2}}{M_{s}^{2}}-\mu_{0} \mathbf{M} \cdot \mathbf{H}-\frac{\mu_{0}}{2} \mathbf{H}_{d}(r) \cdot \mathbf{M}\right] d r
$$

Here $M_{s}=|\mathbf{M}(\mathbf{r})|$ is the spontaneous magnetization, $K_{1}(\mathbf{r})$ denotes the first uniaxial anisotropy constant, $A$ is the exchange stiffness, and $\mathbf{H}$ is the applied magnetic field. Note that Eq. (1) describes a generally random mixture of hard and soft phases but assumes that the crystallites have a common $c$ axis. Physically realized magnetization configurations $\mathbf{M}(\mathbf{r})$ correspond to local or global energy minima, and the hysteresis loop is obtained by tracing the magnetization configuration as a function of the external field $\mathbf{H}$. A key problem is that the magnetostatic self-interaction field $\mathbf{H}_{d}(\mathbf{r})$ is a nonlocal functional of $\mathbf{M}(\mathbf{r})$, which makes it necessary to determine $\mathbf{H}_{d}$ self-consistently. The analysis of the problem shows that it is, in general, not possible to interpret $\mathbf{H}_{d}(\mathbf{r})$ as a local modification of the external field.

An important class of magnetic-reversal phenomena are nucleation processes, which are defined as localized or extended (delocalized) instabilities of a metastable energy 


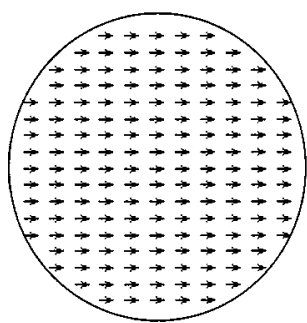

(a)

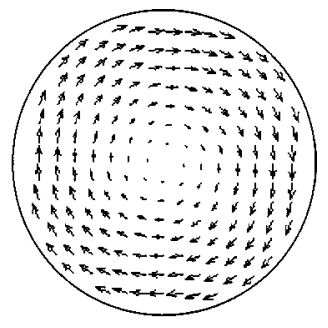

(b)
FIG. 1. Free-surface nucleation modes: (a) coherent rotation, and (b) curling. The figure shows the $x$ and $y$ magnetization deviations in the $x-y$ plane for a sphere (top view on the equatorial plane).

minimum. ${ }^{6,9,10,19,20,22}$ The reverse magnetic field $H_{N}$ at which nucleation occurs is known as the nucleation field: $\mathbf{H}=-H_{N} \mathbf{e}_{z}$. In the simplest case, nucleation occurs at the fully aligned state, where $\mathbf{M}=M_{s} \mathbf{e}_{z}$, and leads to complete magnetic reversal. This corresponds to rectangular hysteresis loops whose coercivity is equal to $H_{N}$. Note, however, that the behavior of the magnet after nucleation goes beyond the scope of nucleation theory, and in practice processes such as domain-wall pinning may inhibit complete reversal.

For some structural models it is possible to obtain exact nucleation fields and nucleation modes. In structurally homogeneous ellipsoids of revolution having an easy magnetization axis (unit vector $\mathbf{e}_{z}$ ) parallel to the axis of revolution there are two exact eigenmodes of interest. ${ }^{10,19,20}$ If the ellipsoid's radius $R$ is smaller than a coherence radius $R_{\text {coh }}$, then the dominating exchange interaction yields coherent (uniform) nucleation [Fig. 1(a)]. For radii larger than $R_{\text {coh }}$ magnetostatic interactions give rise to curling [Fig. 1(b)]. For spheres and long cylinders (magnetized needles) one obtains $R_{\text {coh }}=5.099 \sqrt{A / \mu_{0} M_{s}^{2}}$ and $R_{\text {coh }}=3.682 \sqrt{A / \mu_{0} M_{s}^{2}}$, respectively. As a consequence, $R_{\mathrm{coh}} \approx 10 \mathrm{~nm}$ for a wide range of materials (see also Sec. III C). Note that the nucleation problem is not related to the frequently considered existence of equilibrium domains: hysteresis loops are nonequilibrium phenomena, whereas quantities such as the critical singledomain radius $R_{\mathrm{SD}} \gg R_{\text {coh }}$ refer to equilibrium and describe, for example, ${ }_{6,25}$ the virgin state after thermal demagnetization.

For coherent rotation one obtains the Stoner-Wohlfarth relation

$$
H_{N}=\frac{2 K_{1}}{\mu_{0} M_{s}}+\frac{1}{2}(1-3 D) M_{s},
$$

whereas the curling nucleation field is (see, e.g., Ref. 10)

$$
H_{N}=\frac{2 K_{1}}{\mu_{0} M_{s}}+\frac{c(D) A}{\mu_{0} M_{s} R^{2}}-D M_{s} .
$$

In these equations, $K_{1}$ is the first uniaxial anisotropy constant, and the factor $c$ equals 8.666 for spheres $\left(D=\frac{1}{3}\right)$ and 6.780 for long cylinders $(D=0)$. In a sense, Eqs. (2) and (3) epitomize the progress in analytic micromagnetics after the seminal domain-wall calculations by Bloch and Landau. It is worthwhile noting that coherent rotation and curling are the only nucleation modes in not-too-elongated homogeneous ellipsoids of revolution. ${ }^{10}$ Localized modes, where the nucle- ation process occurs in a small subvolume of the magnet, is unfavorable from the point of view of exchange energy, whereas the buckling mode can be excluded for aspect ratios smaller than 4.6.

This paper consists of two parts. In Sec. II we report a novel nucleation mode, denoted here as bulging, and in Sec. III we interpret this mode in terms of demagnetizing-field contributions.

\section{MAGNETIZATION BULGING}

In homogeneous, single-phase magnets there are only two modes, namely coherent rotation and curling, but this is not necessarily the case in two-phase nanostructures. Here we deal with comparatively large soft or semihard inclusions, which have a disproportionally strong influence on the coercivity of real magnets. We disregard extremely small soft (or semihard) regions, which are ideally exchange-coupled to the hard matrix and yield a micromagnetically homogeneous material characterized by a volume-averaged anisotropy constant $\langle K\rangle .^{1}$ In this "plateau" or "virtual crystal" regime, the nucleation modes are delocalized, that is they extend throughout the magnet. For ellipsoidal magnet shapes the corresponding nucleation mode is a curling-like but in general perturbed by demagnetizing-field inhomogeneities. By comparison, extended soft regions give rise to difficult-totreat localized modes. ${ }^{9}$ As a model, we consider a semihard or soft ferromagnetic sphere of magnetization $M_{s}$, surrounded by and exchange-coupled to a very hard surface layer of fixed magnetization $M_{s} \mathbf{e}_{z}$. This case is not only scientifically interesting but also of practical interest in twophase nanomagnetism, because soft regions are often embedded in a more or less aligned hard matrix.

\section{A. Boundary conditions}

The calculations leading to Eqs. (1) and (2) are based on the assumption of free-surface boundary conditions $\mathbf{n} \cdot \nabla \mathbf{M}$ $=0$. In two-phase structures, such as magnetic multilayers ${ }^{1}$ and composite oxide particles, ${ }^{26}$ the exchange coupling modifies the boundary conditions at the interfaces. Starting from the boundary-condition analysis by Skomski and Coey ${ }^{1}$ it is straightforward to show that the general interface boundary conditions involving $A$ then reduce to clamped boundary conditions $\mathbf{M}=M_{s} \mathbf{e}_{z}$. Physically, this means that the nucleation mode remains localized in the soft phase (compare Sec. III A). Furthermore, a very hard shell suppresses micromagnetic surface modes such as the ones considered by Suhl and Bertram. $^{17}$

\section{B. Angular dependence}

To calculate the nucleation fields we start from the method summarized in Ref. 10. Essentially, one must write down the differential equation for the perpendicular magnetization mode $\mathbf{m}(\mathbf{r})=\mathbf{M}(\mathbf{r})-M_{s} \mathbf{e}_{z}$ and find the eigenmodes $\mathbf{m}(\mathbf{r})$ of that equation. Here we consider two modes: curlingtype modes, where ${ }^{10}$

$$
\mathbf{m}(r)=m(r, \theta)\left(\cos \phi \mathbf{e}_{y}-\sin \phi \mathbf{e}_{x}\right)
$$

and a "quasicoherent" or "purely radial" mode 


$$
\mathbf{m}(\mathbf{r})=m(r) \mathbf{e}_{m},
$$

where $\mathbf{e}_{z} \cdot \mathbf{e}_{m}=0$. In both cases, the magnitude of the mode is given by $m(\mathbf{r})=|\mathbf{m}(\mathbf{r})|$.

To prove that Eqs. (4a) and (4b) are exact nucleation modes, rather than trial functions, we have to show that they are eigenfunctions of the corresponding differential equations. This includes the calculation of the radial eigenfunctions $m(r)$. As outlined in Ref. 10, the differential equations are obtained by minimizing the total magnetic energy Eq. (1) with respect to the small quantity $\mathbf{m}(\mathbf{r})$. For the curling and radial modes we obtain

$$
-A \nabla^{2} m+2 K_{1} m+\mu_{0} H M_{s} m-\frac{\mu_{0}}{3} M_{s}^{2} m=0
$$

and

$$
-A \nabla^{2} m+2 K_{1} m+\mu_{0} H M_{s} m=0,
$$

respectively. Aside from the different boundary conditions, and aside from minor variations in the representation, Eq. (5a) is a well-known expression. ${ }^{10,20}$ The magnetostatic term $-\mu_{0} m M_{s}^{2} / 3$ reflects the flux-closure clearly visible in Fig. 1(b).

Equation (5b) is a generalization of the coherent-rotation limit $2 K_{1} m+\mu_{0} H M_{s} m=0$. An interesting feature of Eq. (5b) is the absence of magnetostatic self-interaction terms. For coherent rotation, that is for $\mathbf{m}(\mathbf{r})=\mathbf{m}_{0}$, the argument is trivial: since the magnetostatic energy of a homogeneously magnetized sphere is independent of the magnetization direction, it can be incorporated into a physically unimportant zero-field energy (compare Sec. 9.2.1 in Ref. 10). To analyze the magnetostatic energy of an arbitrary quasicoherent configuration $\mathbf{m}(\mathbf{r})=\mathbf{m}(r)$ we divide the spherical magnet into infinitesimally small shells characterized by $\mathbf{m}_{i}=\mathbf{m}\left(\mathbf{r}_{i}\right)$. The total magnetostatic self-interaction energy $E_{\mathrm{ms}}$ then decomposes into interactions between pairs of shells, $E_{\mathrm{ms}}$ $=\sum_{i>j} E_{\mathrm{ms}}(i, j)$. Taking into account that the magnetizations of the shells depend on $r$ only, but not on $\theta$ and $\phi$, and utilizing the angular symmetry of the dipolar interaction we find that $E_{\mathrm{ms}}(i, j)=0$ for any pair of shells, and therefore $E_{\mathrm{ms}}=0$.

\section{Radial eigenfunctions}

Rewriting Eq. (5) in terms of spherical coordinates and putting $m(r, \theta)=F(r) \Theta_{n}(\theta)$ yields the radial equation

$$
\frac{d^{2} F}{d r^{2}}+\frac{2}{r} \frac{d F}{d r}+\left(k^{2}-\frac{n(n+1)}{r^{2}}\right) F=0 .
$$

In the case of curling, $n=1$ and

$$
k^{2}=-\frac{2 K_{1}+\mu_{0} M_{s} H-\mu_{0} M_{s}^{2} / 3}{2 A},
$$

whereas the quasicoherent case is characterized by $n=0$ and

$$
k^{2}=-\frac{2 K_{1}+\mu_{0} M_{s} H}{2 A} .
$$

The angular eigenfunctions are $\Theta_{0}(\theta)=1$ and $\Theta_{1}(\theta)$ $=\sin (\theta)$, respectively. The solutions

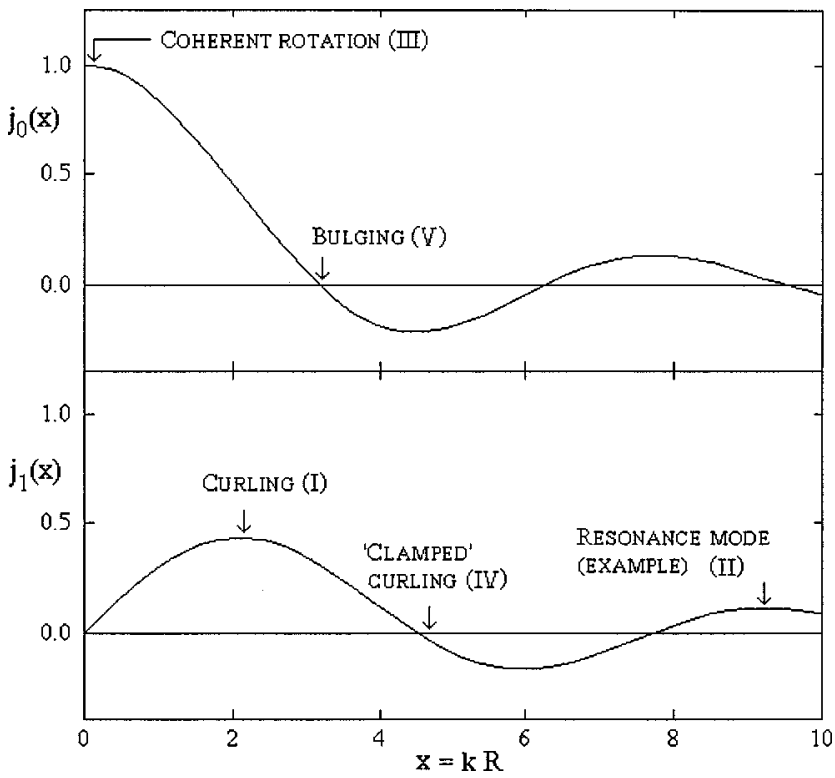

FIG. 2. Spherical Bessel functions and their micromagnetic interpretation.

of Eq. (7) are spherical Bessel functions: $F(r)=j_{0}(k r) .{ }^{27}$ In particular, $j_{0}(x)=\sin (x) / x$ describes purely radial modes and $j_{1}(x)=\sin (x) / x^{2}-\cos (x) / x$ describes curling-type modes.

The final step is to incorporate the boundary conditions. Free boundary condition correspond to $d j_{n}(k r) / d r=0$ at $r$ $=R$. Curling is realized for $x=k R=2.0816,{ }^{10}$ corresponding to point (I) in Fig. 2. Putting $k=2.0816 / R$ into Eq. (7a) then reproduces the spherical limit $\left(D=\frac{1}{3}\right)$ of Eq. (3). It is important to note that the field must be negative (reversed) to yield the right sign of $k^{2}$. Other maxima, such as point (II) in Fig. 2 , also satisfy the boundary condition $d j_{1} / d x=0$. However, the additional oscillations enhance the exchange energy, correspond to more negative fields, and have no physical meaning in the context of nucleation (see p. 216 in Ref. 10).

\section{Bulging vs. coherent rotation}

A trivial example of a purely radial mode is the coherent mode, where $d j_{0} / d x=0$. In terms of Eq. (7b), the coherent mode is reproduced by $k=0$ and corresponds to point (III) in Fig. 2. This implies $j_{0}(k r)=1$ (no radial variation), and the nucleation field is equal to $2 K_{1} / \mu_{0} M_{s}$. Note that putting $k$ $=0$ satisfies the free boundary conditions $d j_{n} / d x=0$ for any value of $n$, but only for $n=0$ this corresponds to a nonzero mode.

To realize clamped boundary conditions we have to ensure that $j_{n}(k R)=0$, rather than $\left.\left(d j_{n} / d x\right)\right|_{k R=0}$. This yields two nucleation modes. Aside from a modified curling mode (Sec. II E), there is an incoherent mode chararacterized by a quasi-coherent (purely radial) angular dependence. In Fig. 2, this corresponds to point $(\mathrm{V})$. The novel mode, which we will call bulging, is shown in Fig. 3(a). It is characterized by the nucleation field

$$
H_{N}=\frac{2 K_{1}}{\mu_{0} M_{s}}+2 \pi^{2} \frac{A}{\mu_{0} M_{s} R^{2}} .
$$

Bulging processes in aspherical ellipsoids are more difficult to calculate, because the abovementioned argument regard- 


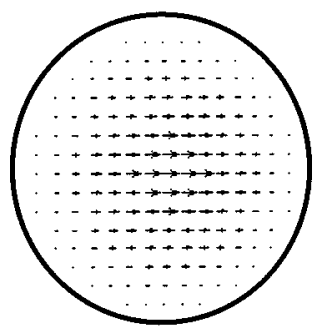

(a)

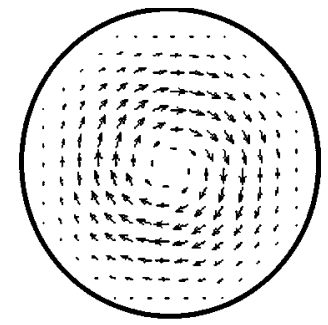

(b)
FIG. 3. Clamped nucleation modes: (a) bulging and (b) modified curling. The figure shows the $x$ and $y$ magnetization deviations in the $x-y$ plane (top view on the equatorial plane).

ing the magnetostatic self-energy does not apply. However, from the qualitative behavior of ellipsoidal wave functions we expect modes similar to Fig. 3(a).

It is interesting to compare the bulging nucleation field with the coherent-rotation nucleation field (anisotropy field) $2 K_{1} / \mu_{0} M_{s}$, which is obtained by putting $D=\frac{1}{3}$ in Eq. (2). Unlike the coherent-rotation nucleation field, the bulging nucleation field depends on the size of the semihard or soft inclusion: it is highest for small inclusions (Sec. III A).

\section{E. Modified curling}

The condition $j_{1}(k R)=0$ yields a "clamped" curling mode with a modified radial dependence [Fig. 3(b) and point (IV) in Fig. 2]. The corresponding nucleation field is

$$
H_{N}=\frac{2 K_{1}}{\mu_{0} M_{s}}-\frac{1}{3} M_{s}+40.382 \frac{A}{\mu_{0} M_{s} R^{2}} .
$$

The transition between bulging and clamped curling occurs at $R_{\text {coh }}=7.869 \sqrt{A / \mu_{0} M_{s}^{2}}$, which is somewhat larger than for free-surface nucleation.

For curling in long cylinders coated by a hard surface layer, the nucleation field is obtained from the first zero of the Bessel function $J_{1}(x)$, which occurs at $x=3.83171$. The result is

$$
H_{N}=\frac{2 K_{1}}{\mu_{0} M_{s}}+29.364 \frac{A}{\mu_{0} M_{s} R^{2}}
$$

as compared to Eq. (3) with $c=6.780$ and $D=0$ for uncoated cylinders.

\section{DISCUSSION}

\section{A. Applicability of the model}

A difficult problem regarding the model considered in this work is to what extent a spherical geometry is able to approximate nanostructures encountered in practice. It is, however, possible to discuss the effect of the present model assumptions on the hysteresis loop. The first point is that nanostructures encountered in practice are often isotropic or only partly textured, so that the $K_{1}$ term in Eq. (1) must be replaced by a random anisotropy term (see, Ref. 6). As a consequence, the loop is no longer rectangular and nucleation starts from an incompletely aligned magnetization configuration (compare Ref. 22). A similar effect is caused by demagnetizing-field inhomogeneities (Sec. III B). This means that the present model approximates real hysteresis loops by rectangular loops whose width is given by the nucleation field. The neglect of the real loop shape is important from a quantitative point of view, but it does not invalidate the qualitative features considered in this work.

A more subtle point is the assumption of a "very hard" shell, which leads to the clamped boundary conditions used in Sec. II. The condition "very hard" means that the anisotropy field $2 K_{h} / \mu_{0} M_{s}$ of the hard phase must be much higher than the nucleation field $H_{N}$. From Eq. (8) we see that this condition breaks down for very small inclusions. The clamped boundary conditions, which yield well-localized and easy-to-calculate nucleation modes, must then be replaced by general boundary conditions of the type $A_{h} \partial m_{h} / \partial r=A_{s} \partial m_{s} / \partial r,{ }^{1}$ where the respective indices refer to the hard and soft regions. The point is that the nucleation mode penetrates from the soft phase into the hard phase when the exchange energy density, scaling as $A / R^{2}$, is able to compete against the anisotropy energy density $K_{1} \cdot{ }^{1}$ With decreasing radius $R$, this leads to a $K_{1}$-dependent radial delocalization of the bulging mode until the delocalized $\left\langle K_{1}\right\rangle$ regime is reached. However, neither the plateau itself nor the approach to the plateau are of interest in the present context.

\section{B. Local magnetic fields}

A popular explanation of demagnetizing factors of real materials is in terms of nonuniform local stray fields caused by morphological inhomogeneities and adding to the local anisotropy field $2 K_{1}(\mathbf{r}) / \mu_{0} M_{s}(\mathbf{r})$. In homogeneous ellipsoids of revolution magnetized along the axis of revolution the demagnetizing field is equal to $-D M_{s}$, where $D=D_{\|}$is the demagnetizing factor. From elementary electrodynamics it follows in particular that $D=0$ for long cylinders (needles), $D=\frac{1}{3}$ for spheres, and $D=1$ for oblate thin films. ${ }^{28}$ More generally, in arbitrary ellipsoids the three eigenvalues of the demagnetizing tensor obey $D_{x}+D_{y}+D_{z}$ $=1$, where the subscripts refer to the ellipsoid's principal axes. More generally, according to the Brown-Morrish theorem $^{10}$ the magnetostatic self-energy of any homogeneously magnetized body of arbitrary shape can be written as

$$
\begin{aligned}
E_{m s} & =-\frac{1}{2} \mu_{0} \mathbf{M} \cdot \int \mathbf{H}(\mathbf{r}) d \mathbf{r} \\
& =\frac{\mu_{0}}{2}\left(D_{x} M_{x}^{2}+D_{y} M_{y}^{2}+D_{z} M_{z}^{2}\right) V,
\end{aligned}
$$

where we obey $D_{x}+D_{y}+D_{z}=1$. However, even in this simplified case the local magnetic field is inhomogeneous, and local fields are of great importance in real materials (see, e.g., Refs. 14,18,24,29).

An advantage of the present model is the absence of inhomogeneous magnetostatic fields. In fact, the assumption that the soft inclusion and the hard shell have the same magnetization means that the (magnetostatic) demagnetizing field before nucleation is homogeneous throughout the magnet. It is therefore not possible to ascribe the difference between Eqs. (2) and (9) to any magnetostatic demagnetizing field. The same is true for other "coated" ellipsoids such as the cylinders considered in Sec. II E. 
(C)

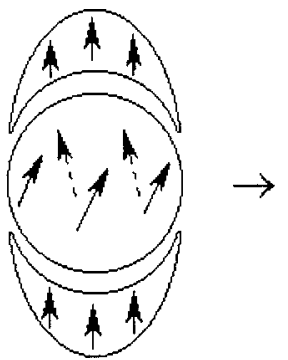

(E)

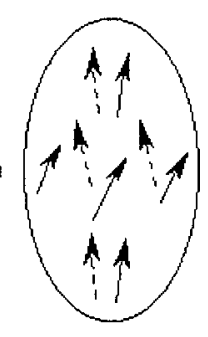

FIG. 4. Limitations of the magnetostatic demagnetizing-field approach. By a gedanken experiment, a prolate ellipsoid (E) is formed from a sphere $(\mathrm{S})$ and two caps $(\mathrm{C})$. The caps give rise to a Maxwellian contribution, but when they touch the sphere, there are also exchange contributions.

\section{The role of exchange}

Exchange interactions are well-known to dominate magnetostatic interactions on small length scales. On an atomic scale, the exchange field, which does not enter Maxwell's equations, is much larger than magnetostatic fields. From relativistic scaling considerations ${ }^{23}$ it follows that magnetostatic interactions become important on a length scale $l_{0}$ $=a_{0} / \alpha=7.252 \AA$ in typical ferromagnetic solids, where $a_{0}$ $=0.5292 \AA$ is the Bohr radius and $\alpha=\frac{1}{137}$ is Sommerfeld's fine structure constant. ${ }^{30}$ This indicates that both magnetostatic and exchange interactions are important in magnetic nanostructures. Furthermore, from an experimental point of view it is difficult to separate magnetostatic and exchange contributions. Both are quadratic in the spontaneous magnetization $M_{s}=|\mathbf{M}|$ and have essentially the same temperature dependence, so that they cannot be distinguished by the temperature-dependent measurement methods ${ }^{3,31}$ usually employed to separate $K_{1}$ contributions from magnetostatic contributions.

Figure 4 illustrates the competition between magnetostatic self-interaction and exchange by a gedanken experiment. A prolate ellipsoid (E) is made by adding two caps (C) to sphere (S). Of course, the two caps yield a magnetostatic demagnetizing-field contribution, which is obtained from Maxwell's equations, but when the caps touch the sphere, then there is also an exchange contribution associated with the required continuity of the magnetization.

\section{Effective demagnetizing factors}

A semiphenomenological way of discussing magnetic reversal is the Kronmüller analysis ${ }^{31}$ based on the equation

$$
H_{N}=\frac{2 K_{1}}{\mu_{0} M_{s}}-D_{\text {eff }} M_{s},
$$

where $D_{\text {eff }}$ is effective demagnetizing factor. (In the sense of Sec. III A, we assume that the nucleation-field $H_{N}$ is equal to the coercivity $H_{c}$ ). Comparing this equation with Eqs. (2), (3), and (8)-(10) yields $D_{\text {eff }}$ as a function of the particle or inclusion radius. Figure 5 shows the result for spherical magnets. We see that the validity of the "magnetostatic" demagnetizing factor $D_{\text {eff }}=D=\frac{1}{3}$ is restricted to free-surface curling in macroscopic magnets $(R \gg 10 \mathrm{~nm})$. In all other cases there is an exchange contribution to $D_{\text {eff }}$. Equations (3) and (8) show that the exchange and magnetostatic contributions are

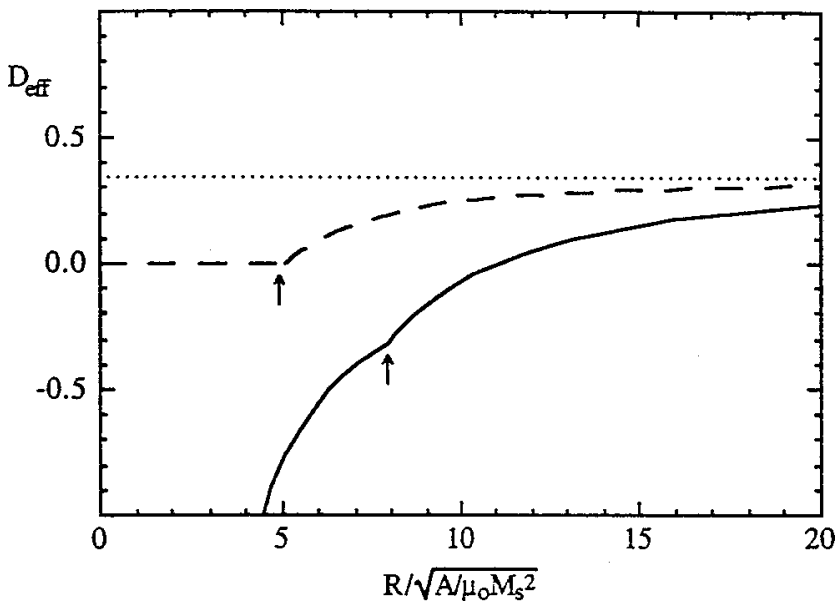

FIG. 5. Effective demagnetizing factors as a function of the sphere radius: Maxwell prediction (dotted line), homogeneous sphere (dashed line), and coated sphere (solid line). For very small radii the assumption of an ideally hard sphere becomes unrealistic. The radius is measured in units of $\sqrt{A / \mu_{0} M_{s}^{2}}$.

of opposite sign, a phenomenon which is indeed observed in practice. ${ }^{3}$ Note also that, for coated spheres, $D_{\text {eff }}$ is negative up to $R_{0}=11.007 \sqrt{A / \mu_{0} M_{s}^{2}}$ (Fig. 5). Below $R_{0}$, the exchange contribution overcompensates the magnetostatic contribution to the effective demagnetizing factor.

\section{E. Hysteresis-loop overskewing}

An important demagnetizing phenomenon is the skewing (shearing) of hysteresis loops (Fig. 6). The shearing procedure is used to realize demagnetizing-field corrections, which account for the nonzero sample-shape dependent demagnetizing fields encountered in open-circuit measurements. The procedure consists in considering skewed reference curves $M(H-D M)$ rather than $M(H)$. However, the experimental aspects of this procedure are by no means trivial. For example, in Ref. 5 the complete neglect of the demagnetizing factor $(D=0)$ gave rise to an unphysically low energy product of about $40 \mathrm{MGOe}$, whereas putting $D$ $=1$, as appropriate for thin films, would yield an overskewing of the hysteresis loop with an extrapolated energy product of more than $60 \mathrm{MG} \mathrm{Oe} \mathrm{(Fig.} \mathrm{6).} \mathrm{Similar} \mathrm{difficulties} \mathrm{are}$ encountered in other magnetic systems. By comparing the magnetization curves of $\mathrm{Ni}$ and $\mathrm{Sm}_{2} \mathrm{Fe}_{17} \mathrm{~N}_{3}$ particles fixed in epoxy resin the experimental demagnetizing factors are $D_{\|}$ $=0.14$ and $D_{\perp}=0.33,{ }^{32}$ so that $D_{\|}+2 D_{\perp}=0.80$ rather than $D_{\|}+2 D_{\perp}=1$.

A popular approach is to ascribe demagnetizing-field irregularities to inhomogeneous magnetic fields naturally occurring in real magnets. However, as discussed in Sec. III C, magnetostatic fields are not the only consideration, and in Sec. III B we saw that there are no inhomogeneous fields in the models considered here. On the other hand, in many cases the nucleation field, and therefore the hysteresis loop, depend on the exchange stiffness $A$ and on the particle radius $R$. The relation between this dependence and the loop overskewing is illustrated in Fig. 7. Essentially, the skewing consists in the replacement of an open-circuit nucleation field $H_{N 1}$ by a closed-circuit nucleation field $H_{N 2}$. A comprehen- 


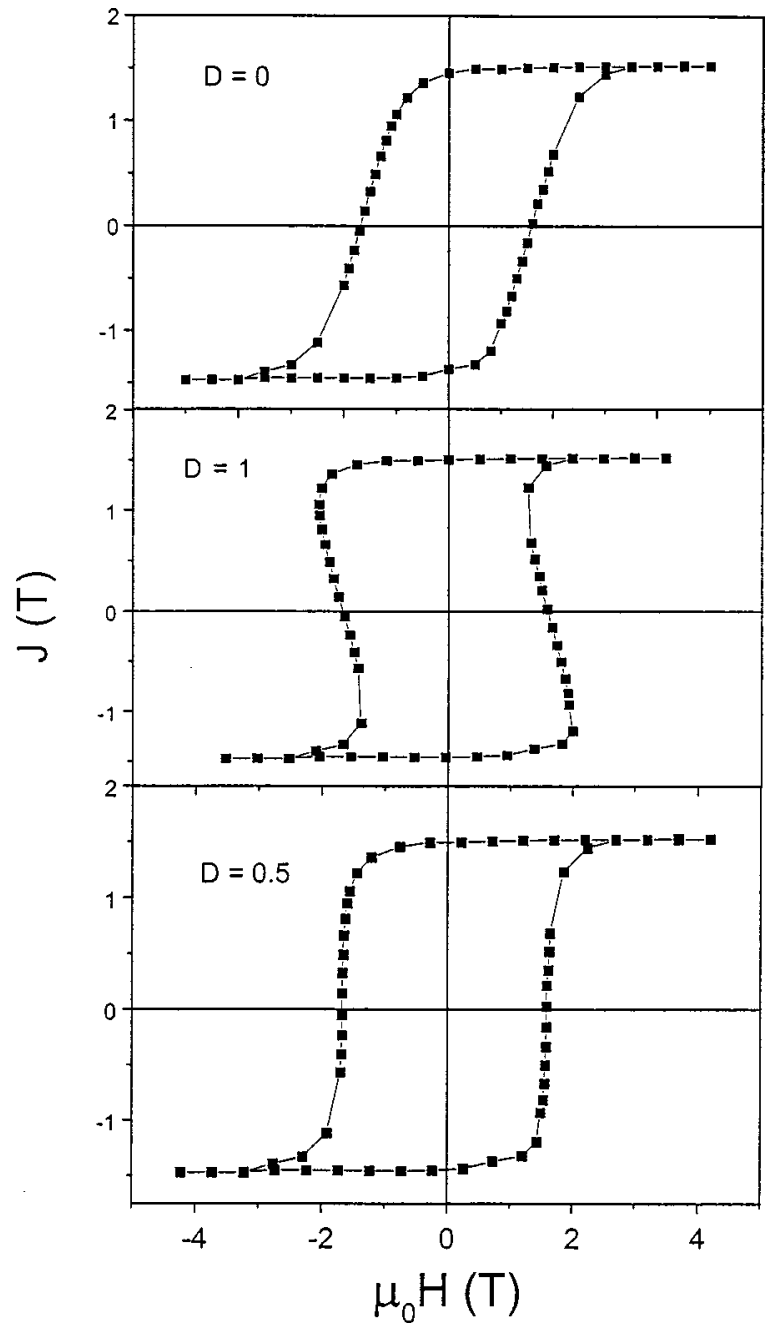

FIG. 6. Demagnetizing-field correction for a Fe/Pt film: (a) raw data, (b) overskewing due to $D=1$, and (c) infinite-slope method $\left(J=\mu_{0} M\right)$. Skewing corrections are of practical importance, because they make it possible to compare the properties of magnets of different shapes.

sive quantitative analysis of the difference $H_{N 2}-H_{N 1}$ goes beyond the scope of this work and will be published elsewhere, but from Fig. 5 and from the nucleation fields presented in Sec. II we see that this difference cannot be reduced to a purely magnetostatic contribution.

It is instructive to compare our qualitative approach with experimental procedures to circumvent the problem of overskewing. To obtain a reasonable $(B H)_{\max }$ value, Liu et al. ${ }^{5}$ used an approximate deskewing procedure based on the assumption of an infinite slope $d M / d H=\infty$ at $H=H_{c}$ (Ref. 33) and obtained $(B H)_{\max }=52.8 \mathrm{MG}$ Oe for $D=0.48$. The approximate character of this method is proven by a simple counter-example: for an ensemble of independent particles having a very broad distribution of coercivities one has $d M / d H>0$ at $H=H_{c}$, independently of the strength of the demagnetizing field. Summarizing, our approach gives a

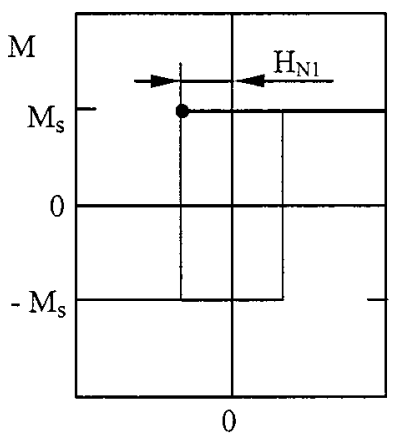

$\mathrm{H}$ (arb. units)

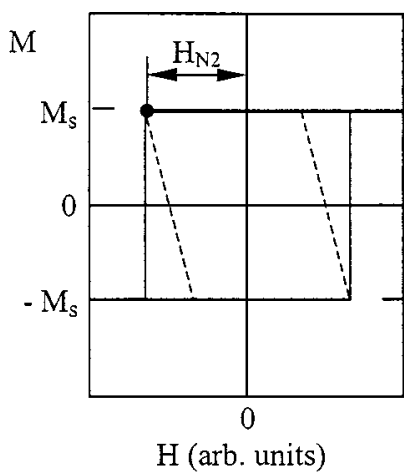

(b)
FIG. 7. Theoretical demagnetizing-field correction: (a) original loop and (b) skewed loop (dashed line). Since overskewing corresponds to an instability, a vertical solid line is used to show the physically reasonable rectangular-loop behavior. The nucleation fields for the different sample shapes are discussed in the main text. From those equations follows that exchange and magnetostatic contributions are of opposite sign.

qualitative correct account of demagnetizing factors in nanomagnets, although a quantitative interpretation of the demagnetizing behavior of real, disordered nanostructures remains a challenge.

An alternative interpretation of the bulging demagnetization factor Eq. (9) is that the hard shell yields an effective demagnetizing field contribution by exchange biasing the soft core. Although meaningful for the bulging mode, this explanation cannot be generalized. For example, the curling nucleation field Eq. (3) depends on the exchange stiffness $A$, but there is no phase or surface contribution that could be interpreted as a source of biasing.

\section{CONCLUSIONS}

The nucleation of reversed domains in two-phase nanostructures is qualitatively different from the situation encountered in structurally homogeneous ellipsoids of revolution. In two-phase magnets consisting of small soft-magnetic or semihard particles surrounded by hard-magnetic shells nucleation is realized by a nucleation mode called bulging. Since the coupling between the soft and hard phases creates a radial inhomogeneity of the magnetization, the bulging mode is incoherent but has the purely radial angular symmetry of the coherent mode. The corresponding effective demagnetizing factors are generally smaller than predicted from Maxwell's equations, because both magnetostatic and exchange fields contribute to the demagnetizing behavior.

\section{ACKNOWLEDGMENTS}

The authors are grateful to Professor S. S. Jaswal for discussing the presentation of the material. This research was supported by NSF, U.S. DOE, AFOSR, and DARPA.
* Author to whom correspondence should be addressed. FAX: (001)-402-472-2879. Electronic address:

rskomski@unlinfo.unl.edu

${ }^{1}$ R. Skomski and J. M. D. Coey, Phys. Rev. B 48, 15812 (1993).

2 J. M. D. Coey, Rare-earth Iron Permanent Magnets (Oxford University Press, Oxford, 1996). 
${ }^{3}$ I. A. Al-Omari and D. J. Sellmyer, Phys. Rev. B 52, 3441 (1995).

${ }^{4}$ D. J. Sellmyer and Z. S. Shan, in Magnetic Hysteresis in Novel Magnetic Materials, edited by G. C. Hadjipanayis (Kluwer, Dordrecht, 1997), p. 419.

${ }^{5}$ J. P. Liu, C. P. Luo, Y. Liu, and D. J. Sellmyer, Appl. Phys. Lett. 72, 483 (1998).

${ }^{6}$ R. Skomski and J. M. D. Coey, Permanent Magnetism (Institute of Physics, Bristol, 1999).

${ }^{7}$ Zh. Fan and Zh. Jinxiu, Phys. Rev. Lett. 75, 2027 (1995).

${ }^{8}$ A. Holz and C. Scherer, Phys. Rev. B 50, 6209 (1994).

${ }^{9}$ R. Skomski, J. Appl. Phys. 83, 6503 (1998).

${ }^{10}$ A. Aharoni, Introduction to the Theory of Ferromagnetism (Oxford University Press, Oxford, 1996).

${ }^{11}$ D. Sander, R. Skomski, C. Schmidthals, A. Enders, and J. Kischner, Phys. Rev. Lett. 77, 2566 (1996).

${ }^{12}$ R. P. Cowburn, J. Ferré, S. J. Gray, and J. A. C. Bland, Phys. Rev. B 58, 11507 (1998).

${ }^{13}$ A. F. Khapikov, Phys. Rev. Lett. 80, 2209 (1998).

${ }^{14}$ T. Schrefl, J. Fidler, and H. Kronmüller, Phys. Rev. B 49, 6100 (1994).

${ }^{15}$ R. Fischer and H. Kronmüller, Phys. Rev. B 54, 7284 (1996).

${ }^{16}$ R. Fischer and H. Kronmüller, J. Magn. Magn. Mater. 184, 166 (1998).

${ }^{17}$ H. Suhl and H. N. Bertram, J. Appl. Phys. 82, 6128 (1997).

${ }^{18}$ T. Schrefl and J. Fidler, J. Magn. Magn. Mater. 177-181, 970 (1998).
${ }^{19}$ A. Aharoni, Rev. Mod. Phys. 34, 227 (1962).

${ }^{20}$ W. F. Brown, Micromagnetics (Wiley, New York, 1963).

${ }^{21}$ T. Leineweber and H. Kronmüller, J. Magn. Magn. Mater. 176, 145 (1997)

${ }^{22}$ A. Aharoni, J. Appl. Phys. 82, 1281 (1997).

${ }^{23}$ R. Skomski, H.-P. Oepen, and J. Kirschner, Phys. Rev. B 58, 3223 (1998).

${ }^{24}$ R. Fischer, T. Leineweber, and H. Kronmüller, Phys. Rev. B 57, 10723 (1998).

${ }^{25}$ R. Skomski, J.-P. Liu, J. M. Meldrim, and D. J. Sellmyer, in Magnetic Anisotropy and Coercivity in Rare-Earth TransitionMetal Alloys, edited by L. Schultz and K.-H. Müller (Werkstoffinformationsgesellschaft, Frankfurt/Main, 1998), p. 277.

${ }^{26}$ A. Aharoni, J. Appl. Phys. 62, 2576 (1987).

${ }^{27}$ Handbook of Mathematical Functions, edited by A. Abramowitz and I. A. Stegun (Dover, New York, 1972).

${ }^{28}$ J. A. Osborn, Phys. Rev. 67, 351 (1945).

${ }^{29}$ J. Dubowik, Phys. Rev. B 54, 1088 (1996).

${ }^{30}$ Aside from a numerical prefactor, $1_{0}$ corresponds to the exchange-length parameter $\sqrt{A / \mu_{0} M_{S}^{2}}$.

${ }^{31}$ H. Kronmüller, K. D. Durst, and M. Sagawa, J. Magn. Magn. Mater. 74, 291 (1988).

${ }^{32}$ K. Kobayashi, X.-L. Rao, J. M. D. Coey, and D. Givord, J. Appl. Phys. 80, 6385 (1996).

${ }^{33}$ C. D. Mee and E. D. Daniel, Magnetic Recording (McGraw-Hill, New York, 1987), Vol. I, p. 385. 